\title{
A potential role for Galectin-3 inhibitors in the treatment of COVID-19
}

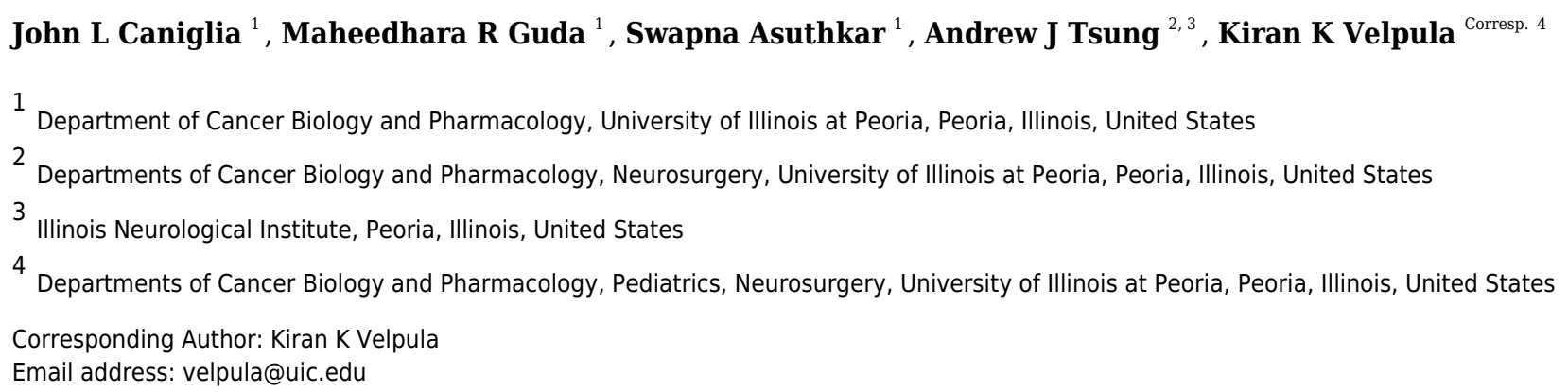

The outbreak of severe acute respiratory syndrome coronavirus 2 (SARS-CoV2), the causative agent of coronavirus disease 2019 (COVID-19), has been declared a global pandemic by the World Health Organization. With no standard of care for the treatment of COVID-19, there is an urgent need to identify therapies that may be effective in treatment. Recent evidence has implicated the development of cytokine release syndrome (CRS) as the major cause of fatality in COVID-19 patients, with elevated levels of IL- 6 and TNF- $\alpha$ observed in patients. Galectin-3 (Gal-3) is an animal lectin that has been implicated in the disease process of a variety of inflammatory conditions. Inhibitors of the small molecule Gal-3 have been shown to reduce the levels of both IL- 6 and TNF- $\alpha$ in vitro and have shown anti-inflammatory effects in vivo. Additionally, a key domain in the spike protein of $\beta$ coronaviridae, a genus which includes SARS-CoV2, is nearly identical in morphology to human Gal-3. These spike proteins are critical for the virus' entry into host cells. Here we provide a systematic review of the available literature and an impetus for further research on the use of Gal-3 inhibitors in the treatment of COVID-19. Further, we propose a dual mechanism by which Gal-3 inhibition may be beneficial in the treatment of COVID-19, both suppressing the host inflammatory response and impeding viral attachment to host cells. 
May $28^{\text {th }} 2020$

To,

Dr. Nikolajs Sjakste,

Academic Editor

Peer J

Dear Dr. Sjakste,

On behalf of all authors, I thank the reviewers for the valuable comments on the literature review by Caniglia et al., and have edited the review to address their concerns. All the comments were addressed in a point-by point manner and now we believe that this literature review is suitable for publication in PeerJ.

If you have any questions or need further information, please feel free to contact me at (309) 6713413 or by e-mail: velpula@uic.edu

\section{Sincerely,}

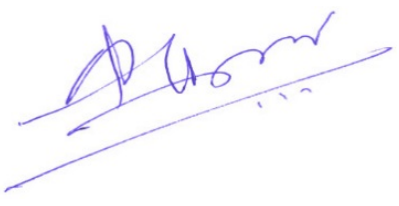

Kiran Velpula

1

2 A Potential Role for Galectin-3 Inhibitors in the Treatment of COVID-19 
3 John L. Caniglia ${ }^{1}$, Maheedhara R. Guda ${ }^{1}$, Swapna Asuthkar ${ }^{1}$, Andrew J. Tsung ${ }^{1,2,4}$, and Kiran K.

4 Velpula ${ }^{1,2,3}$

5

6 Departments of Cancer Biology and Pharmacology ${ }^{1}$, Neurosurgery ${ }^{2}$, Pediatrics ${ }^{3}$, University of

7 Illinois College of Medicine at Peoria; Illinois Neurological Institute ${ }^{4}$, Peoria, IL

\section{Abstract}

The outbreak of severe acute respiratory syndrome coronavirus 2 (SARS-CoV2), the causative agent of coronavirus disease 2019 (COVID-19), has been declared a global pandemic by the World Health Organization. With no standard of care for the treatment of COVID-19, there is an urgent need to identify therapies that may be effective in treatment. Recent evidence has implicated the development of cytokine release syndrome (CRS) as the major cause of fatality in COVID-19 patients, with elevated levels of IL-6 and TNF- $\alpha$ observed in patients. Galectin-3 (Gal-3) is an animal lectin that has been implicated in the disease process of a variety of inflammatory conditions. Inhibitors of the small molecule Gal-3 have been shown to reduce the levels of both IL-6 and TNF- $\alpha$ in vitro and have shown anti-inflammatory effects in vivo. Additionally, a key domain in the spike protein of $\beta$-coronaviridae, a genus which includes SARS-CoV2, is nearly identical in morphology to human Gal-3. These spike proteins are critical for the virus' entry into host cells. Here we provide a systematic review of the available literature and an impetus for further research on the use of Gal-3 inhibitors in the treatment of COVID-19. Further, we propose a dual mechanism by which Gal-3 inhibition may be beneficial in the treatment of COVID-19, both suppressing the host inflammatory response and impeding viral attachment to host cells.

\section{Introduction}

With the ongoing pandemic attributed to the pathogenic SARS-CoV2, there is an urgent need to identify effective treatment options (Zhou et al., 2020). Numerous treatments, most notably the antiviral agent remdesivir and the antiparasitic drug chloroquine, have been extensively studied with inconclusive results (Zhai et al., 2020; Ko et al., 2020). The current absence of a proven, effective anti-viral therapy has resulted in anti-inflammatory agents such as interleukin-6 (IL-6) and tumor necrosis factor alpha (TNF- $\alpha$ ) inhibitors being proposed to mitigate symptoms (Perricone et al., 2020). The rationale is largely based on the finding that 
patients requiring intensive care hospitalization showed highly elevated levels of these proinflammatory cytokines (Perricone et al., 2020). Cytokine inhibitors are currently in clinical trials and have not yet been proven effective.

The lack of an effective standard of care and rate at which COVID-19 is spreading has accelerated the need to identify novel treatment options. Though prior research has elucidated the structural homology between the spike proteins of $\beta$-coronaviruses and human Gal-3, there is no published literature to date referencing this information in the context of SARS-CoV2. Furthermore, there are no articles to date proposing Gal-3 inhibition as a potentially viable treatment to mitigate the entry of SARS-CoV2 and the inflammatory response associated with infection. As such, the authors see a need to spread awareness of the promising indications for targeting Gal-3 in the treatment of COVID-19. This article is intended for all researchers studying galectins and/or SARS-CoV2 but may be particularly beneficial to those focused on drug discovery and development.

\section{Survey Methodology}

\section{Eligibility Criteria}

The following is comprised of original studies that provided information about $\beta$ coronaviridae, Gal-3, or Gal-3 inhibitors. This article includes results from both in vivo and in vitro studies and this information specified where applicable to ensure clarity. The following types of studies were excluded: (1) studies with only an abstract or no full-text available; (2) books, conference papers, and theses.

\section{Search Methodology}

To retrieve primary literature, electronic searches were performed on PubMed and Google Scholar. A list of search terms can be seen in Table S1. Gal-3 expression was obtained from The Human Protein Atlas with the following query: 'Galectin-3'. The structures of the SARS-CoV2 S1-NTD and Gal-3 were obtained from the RCSB Protein Data Bank (PDB) using the following queries: 'SARS-CoV2 spike protein;' 'Galectin-3.'

\section{Risk of Bias}


66

67

68

To minimize the risk of erroneous conclusions, all authors assessed the cited studies for quality. To address key claims in the article including but not limited to: coronavirus mechanisms of entry, Gal-3 suppression inhibiting IL- 6 and TNF- $\alpha$ release, the side effects of Gal-3 inhibition in humans, and the structural similarities of Gal-3 with the S1-NTD of $\beta$ coronaviruses, multiple sources were cited throughout to mitigate error. Additionally, the systematic use of open-ended search queries ensured that a comprehensive profile of results was yielded on the subject matter.

\section{Galectins and the SARS-CoV2 Spike Protein}

Galectins, a structurally similar family of animal lectins with chemokinetic properties, have been implicated extensively in the immune response (Elola et al., 2018). Gal-3, arguably the most well studied of the galectins, has been shown to activate the pro-inflammatory transcription factor NF-kB and induce the release of both IL-6 and TNF- $\alpha$ (Filer et al., 2009; Uchino et al., 2018). Additionally, data obtained from The Human Protein Atlas shows baseline Gal-3 protein expression in healthy tissues is highest in the lungs, followed by the gastrointestinal tract (stomach, duodenum, small intestine, colon, and rectum) and brain (cortex and hippocampus). This is particularly noteworthy as an increasing number of patients infected with SARS-CoV2 have reported gastrointestinal symptoms such as diarrhea, nausea, vomiting, and abdominal pain (Patel et al., 2020). Additionally, a Mao et. al study assessed 214 cases of COVID-19 in Wuhan, China and concluded that $36.4 \%$ of patients in this cohort exhibited neurological symptoms such as cerebrovascular events, impaired consciousness, and muscle injury (Mao et al., 2020). Additionally, a systematic review from Whittaker et. al reports incidences of headache, seizures, asomnia, and an isolated case of Guillan-Barre syndrome that have occurred following SARS-CoV2 infection (Whittaker, Anson \& Harky, 2020).

The spike proteins found in the $\beta$-genus of coronaviridae share unique structural similarities with human Gal-3 (Li, F., 2016). Structural analysis of the N-terminal domain (NTD) of the spike protein subunit $\mathrm{S} 1$ in murine hepatitis virus (MHV) showed a nearly identical topology to human Gal-3, with the only difference being two additional $\beta$-strands in one of the $\beta$ sheet layers of MHV S1-NTD (Li, F., 2015; Peng et al., 2011) Additionally, a study of bovine coronavirus $(\mathrm{BCoV})$ found significant overlap between the virus's S1-NTD receptor binding domain and the galactose-binding domain of human galectins, strongly supporting a functional similarity (Peng et al., 2012). Pertinent to these findings is the high degree of structural 
97 conservation in the S1-NTD observed amongst the $\beta$-genus of coronaviridae, which now 98 includes SARS-CoV2 (Li, F., 2015). Given this structural similarity, it may be possible that inhibitors against human galectins also have the capability to bind the S1-NTD of $\beta$ coronaviridae. Further research will be required to fully examine this hypothesis. Specifically, the intramolecular forces present within the SARS-CoV2 S1-NTD, such as charge-charge interactions and hydrophobicity, and how they compare to human Gal-3 warrants further investigation. The structures of the SARS-CoV2 S1-NTD and human Gal-3 are shown in Fig. 1 below.

\section{Coronavirus Attachment: Significance of the Galectin-like S1-NTD}

The recognition and binding of membrane-bound cell receptors is the first step in viral infection and a necessary event prior to cell invasion (Blaas, 2016). In $\beta$-coronaviridae such as SARS-CoV2, this function is mediated entirely by the $\mathrm{S} 1$ subunit of the spike protein (Li, F., 2016). The S1 subunit can be further divided into two distinct domains: the NTD and the Cterminal domain (CTD) (Wang, Q. et al., 2020). Though both domains play a role in the adhesion process, the receptor binding mechanisms amongst these viruses can be thought of as predominantly CTD or NTD mediated. A general rule is that the CTD mainly binds peptides while the NTD mainly binds extracellular sugars, though there are exceptions such as that seen in the entry mechanism of MHV via carcinoembryonic antigen cell adhesion molecule 1 (CEACAM1) (Li, F., 2015). A pivotal study by Wang et. al has shown structural evidence that SARS-CoV2 binds to host angiotensin-converting-enzyme-2 (ACE2) receptors in a CTD mediated fashion (Li, F., 2016). The main binding receptors utilized by each of the $\beta$ coronaviridae can be seen in Table 1 below.

As can be seen above, the strongly related virus $\mathrm{BCoV}$ attaches to sialic acids on host cells, specifically 9-O-acetyl-sialic acid (9-O-Ac-Sia), in an NTD mediated mechanism (Peng et al., 2012). Specifically, members of this species known to infect humans such as human coronavirus OC43 (HCoV-OC43) and HKU1 (HCoVHKU1) are among the viruses that bind 9$O$-Ac-Sia (Tortorici et al., 2019). In humans, sialic acids including 9-O-Ac-Sia are most present within the body at mucosal surfaces such as the nasopharynx, lungs, and gastrointestinal tract (Barnard et al., 2019). 
More pertinent than these findings, however, is the evidence that coronaviruses which

130

131

132

133

134

135

136

137

138

139

140

141

142

143

144

145

146

147

148

149

150

151

152

153

154

155

156

157

158

159

160

bind receptors in a CTD-mediated fashion still are reliant upon their galectin-like NTD for functioning. In a study by Wi et. al, it was shown that MERS-CoV, in addition to binding dipeptidyl peptidase 4 (DPP4) through its CTD domain, selectively binds to sialic acids at the NTD domain (Li, W. et al., 2017). Additionally, the depletion of sialic acids through treatment with neuraminidase inhibitors inhibited MERS-CoV entry into Calu-3 human airway cells, demonstrating that sialoconjugate binding by the galectin-like NTD is an essential component of MERS-CoV infection (Li, W. et al., 2017).

To date, there are no studies investigating whether sialic acid binding is an essential component of SARS-CoV2 infection. However, a recent study has elucidated the crystalline structure of the SARS-CoV2 S1-NTD (Ou et al., 2020). Molecular dynamic simulations of the tip of the S1-NTD (amino acids 100-175) reveal a strong interaction with GM1 ganglioside, a molecule commonly found on cell surfaces (Fantini et al., 2020). This data strongly supports a dual attachment model for SARS-CoV2 similar to the mechanism observed in MERS-CoV, where the CTD domain is involved in ACE-2 receptor recognition and the NTD region binds gangliosides on the cell surface to stabilize viral adhesion (Fantini et al., 2020). Human galectins have also been shown to bind GM1 ganglioside with high affinity (Wu et al., 2016). The proposed mechanism by which Gal-3 inhibitors may disrupt SARS-CoV2 attachment is shown in Fig. 2 below.

\section{Galectin-3 Inhibitors in Inflammation}

In addition to Gal-3 inhibition potentially being able to bind and disrupt the NTD of $\beta$ coronaviridae, inhibiting Gal-3 has shown numerous anti-inflammatory effects that may be beneficial in the treatment of COVID-19 (Stegmayr et al., 2019). Retrospective studies of the MERS-CoV and SARS-CoV outbreaks have provided evidence that CRS-induced pneumonia was the major cause of fatality in affected patients (Channappanavar, Perlman, 2017). In SARS$\mathrm{CoV}$, the virus efficiently invades monocytes and dendritic cells, inducing the release of proinflammatory cytokines such as interleukin 1 (IL-1), IL-6, and TNF- $\alpha$ (Law et al., 2005). Recent evidence has implicated the CRS as a major cause of fatality in COVID-19 patients as well, and this process is likely dendritic cell mediated (Moore, June, 2020). A study by Chen et. al has shown that Gal-3 inhibition simultaneously reduces the production of inflammatory cytokines

Peer] reviewing PDF | (2020:05:48942:1:1:NEW 28 May 2020) 
161 such as IL-1 and IL-6 while also increasing the levels of the anti-inflammatory interleukin 10

162 (IL-10) in human dendritic cells (Chen, S. S. et al., 2015). A reduction in IL-1, IL-6, and TNF- $\alpha$

163 levels upon treatment with the Gal-3 inhibitor GB1107 was also seen in an inflammatory model

164 of spinal cord injury (Ren et al., 2019). Treatment with Gal-3 inhibitors shows promise in

165 reducing the incidence of CRS in SARS-CoV2 patients through directly suppressing the release

166 of pro-inflammatory cytokines by dendritic cells. Additionally, as increased Gal-3 levels have

167 been shown in virally infected cells, these inhibitors may preferentially bind in highly affected

168 regions of the body (Wang, W. H. et al., 2019). The proposed mechanism of Gal-3 inhibitors in 169 reducing inflammatory cytokine release can be seen in Fig. 3 below.

170

There are studies that have more specifically examined the effects of Gal-3 inhibition as it relates to viral pathogenesis. A study from Chen et. al has shown that Gal-3 is upregulated in the lungs of mice infected with H5N1 influenza A virus (Chen, Y. J. et al., 2018). Compared to controls, Gal-3 K/O mice had a higher survival rate, with $36 \%$ of Gal-3 K/O mice surviving infection compared to $0 \%$ with Gal-3 expression (Chen, Y. J. et al., 2018). The Gal-3 K/O mice also exhibited significantly lower levels of IL-1 $\beta$ than controls (Chen, Y. J. et al., 2018). Additionally, in a murine study $\gamma$-herpesvirus MHV68, Gal-3 K/O mice mounted a stronger

178 CD8+ T-cell response and showed better viral control compared to controls (Kaur et al., 2018) However, as the role of Gal-3 in regulating the innate immune response is widespread, further studies will be needed to fully determine the systemic effects of Gal-3 inhibition. in clinical trials (Blanchard et al., 2014). The use of the Gal-3 inhibitor TD139 has completed phase I/IIa trials in the treatment of idiopathic pulmonary fibrosis (IPF), which showed the drug to be safe and well tolerated (Saito et al., 2019). A phase IIb trial of the drug is ongoing (Saito et al., 2019). Phase II trials of another Gal-3 inhibitor, belapectin (also known as GR-MD-02), showed a significant reduction in portal hypertension and the development of new esophageal varices (EV) in patients with nonalcoholic steatohepatitis (NASH) complicated by EV (Chalasani et al., 2020). Phase III trials of this drug in the treatment of NASH are currently ongoing. It is worth noting that biweekly infusions of belapectin $(2 \mathrm{mg} / \mathrm{kg})$ over the course of a year did not result in any serious adverse effects (Chalasani et al., 2020). However, given the role 191 of Gal-3 in immunomodulation, more studies will be needed to fully evaluate the safety of 
192 belapectin, as to date it has been administered to just over 3,000 patients. On a similar note, a

193

194

195

196

197

198

199

200

201

202

203

204

205

206

207

208

209

210

211

212

213

214

215

216

217

218

219

220

221 novel galectin-1 inhibitor is currently being developed for the treatment of COVID-19. The drug may be able to modulate the cytokine storm associated with COVID-19 as well as impede viral attachment. However, further studies will be necessary to fully evaluate the proposed drug's efficacy in COVID-19 treatment.

\section{Conclusions}

In summary, Gal-3 is a lectin secreted by many types of cells that exhibits potent proinflammatory effects. This includes inducing the production of IL- 6 and TNF- $\alpha$, cytokines which have been shown to play a critical role in the development of CRS (Filer et al., 2009; Uchino et al., 2018; Stegmayr et al., 2019). Importantly, the spike proteins utilized by $\beta$-coronaviridae show strikingly similar morphology to Gal-3 and exhibit similar sugar-binding capabilities (Li, F., 2016). Taken together, the strong correlation of organs showing high Gal-3 expression and symptoms of SARS-CoV2, anti-inflammatory effects of Gal-3 inhibition, and theorized ability of galectin inhibitors to impair NTD-mediated viral attachment make Gal-3 an attractive potential target in the treatment of COVID-19 (Li, W. et al., 2017; Moore, June, 2020). This treatment may exhibit a dual benefit in both inhibiting viral attachment and reducing the host inflammatory response (Ou et al., 2020; Moore, June, 2020). Further research into the role of extracellular sialic acids in SARS-CoV2 attachment is necessary to fully clarify the role of Gal-3 inhibition as antiviral therapy.

Acknowledgments: The authors thank Mark Linder Walk for the Mind, Illinois Neurological Institute, OSF foundation, Peoria, IL, and KB Strong Foundation, Washington, IL for their support. The authors thank Erika Sung for help in the formatting the manuscript.

\section{References}

Barnard KN, Wasik BR, LaClair JR, Buchholz DW, Weichert WS, Alford-Lawrence BK, Aguilar HC, Parrish CR. 2019. Expression of 9-O- and 7,9-O-Acetyl Modified Sialic Acid in Cells and Their Effects on Influenza Viruses. mBio, 10:10.1128/mBio.02490-19.

Blaas D. 2016. Viral entry pathways: the example of common cold viruses. Wiener medizinische Wochenschrift (1946), 166:211-226. 
222 Blanchard H, Yu X, Collins PM, Bum-Erdene K. 2014. Galectin-3 inhibitors: a patent review

223 (2008-present). Expert opinion on therapeutic patents, 24:1053-1065.

224

225

226

227

228

229

230

231

232

233

234

235

236

237

238

239

240

241

242

243

244

245

246

247

248

249

250

251

252

253

254

255

256

Chalasani N, Abdelmalek MF, Garcia-Tsao G, Vuppalanchi R, Alkhouri N, Rinella M, Noureddin M, Pyko M, Shiffman M, Sanyal A, Allgood A, Shlevin H, Horton R, Zomer E, Irish W, Goodman Z, Harrison SA, Traber PG, Belapectin (GR-MD-02) Study Investigators. 2020. Effects of Belapectin, an Inhibitor of Galectin-3, in Patients With Nonalcoholic Steatohepatitis With Cirrhosis and Portal Hypertension. Gastroenterology, 158:1334-1345.e5.

Channappanavar R, Perlman S. 2017. Pathogenic human coronavirus infections: causes and consequences of cytokine storm and immunopathology. Seminars in immunopathology, 39:529539.

Chen SS, Sun LW, Brickner H, Sun PQ. 2015. Downregulating galectin-3 inhibits proinflammatory cytokine production by human monocyte-derived dendritic cells via RNA interference. Cellular immunology, 294:44-53.

Chen YJ, Wang SF, Weng IC, Hong MH, Lo TH, Jan JT, Hsu LC, Chen HY, Liu FT. 2018. Galectin-3 Enhances Avian H5N1 Influenza A Virus-Induced Pulmonary Inflammation by Promoting NLRP3 Inflammasome Activation. The American journal of pathology, 188:10311042.

Elola MT, Ferragut F, Mendez-Huergo SP, Croci DO, Bracalente C, Rabinovich GA. 2018. Galectins: Multitask signaling molecules linking fibroblast, endothelial and immune cell programs in the tumor microenvironment. Cellular immunology, 333:34-45.

Fantini J, Di Scala C, Chahinian H, Yahi N. 2020. Structural and molecular modelling studies reveal a new mechanism of action of chloroquine and hydroxychloroquine against SARS-CoV-2 infection. International journal of antimicrobial agents:105960.

Filer A, Bik M, Parsonage GN, Fitton J, Trebilcock E, Howlett K, Cook M, Raza K, Simmons DL, Thomas AM, Salmon M, Scheel-Toellner D, Lord JM, Rabinovich GA, Buckley CD. 2009. Galectin 3 induces a distinctive pattern of cytokine and chemokine production in rheumatoid synovial fibroblasts via selective signaling pathways. Arthritis and Rheumatism, 60:1604-1614.

Kaur M, Kumar D, Butty V, Singh S, Esteban A, Fink GR, Ploegh HL, Sehrawat S. 2018. Galectin-3 Regulates gamma-Herpesvirus Specific CD8 T Cell Immunity. iScience, 9:101-119.

Ko WC, Rolain JM, Lee NY, Chen PL, Huang CT, Lee PI, Hsueh PR. 2020. Arguments in favour of remdesivir for treating SARS-CoV-2 infections. International journal of antimicrobial agents, 55:105933.

Law HK, Cheung CY, Ng HY, Sia SF, Chan YO, Luk W, Nicholls JM, Peiris JS, Lau YL. 2005. Chemokine up-regulation in SARS-coronavirus-infected, monocyte-derived human dendritic cells. Blood, 106:2366-2374. 
257 Li F. 2016. Structure, Function, and Evolution of Coronavirus Spike Proteins. Annual review of 258 virology, 3:237-261.

259 Li F. 2015. Receptor recognition mechanisms of coronaviruses: a decade of structural studies.

260 Journal of virology, 89:1954-1964.

261 Li W, Hulswit RJG, Widjaja I, Raj VS, McBride R, Peng W, Widagdo W, Tortorici MA, van

262 Dieren B, Lang Y, van Lent JWM, Paulson JC, de Haan CAM, de Groot RJ, van Kuppeveld

263 FJM, Haagmans BL, Bosch BJ. 2017. Identification of sialic acid-binding function for the

264 Middle East respiratory syndrome coronavirus spike glycoprotein. Proceedings of the National

265 Academy of Sciences of the United States of America, 114:E8508-E8517.

266 Mao L, Jin H, Wang M, Hu Y, Chen S, He Q, Chang J, Hong C, Zhou Y, Wang D, Miao X, Li

267 Y, Hu B. 2020. Neurologic Manifestations of Hospitalized Patients With Coronavirus Disease

2682019 in Wuhan, China. JAMA neurology.

269 Moore JB, June CH. 2020. Cytokine release syndrome in severe COVID-19. Science (New York, 270 N.Y.), 368:473-474.

271 Ou X, Liu Y, Lei X, Li P, Mi D, Ren L, Guo L, Guo R, Chen T, Hu J, Xiang Z, Mu Z, Chen X, 272 Chen J, Hu K, Jin Q, Wang J, Qian Z. 2020. Characterization of spike glycoprotein of SARS-

273 CoV-2 on virus entry and its immune cross-reactivity with SARS-CoV. Nature communications, 274 11:1620-020-15562-9.

275 Patel KP, Patel PA, Vunnam RR, Hewlett AT, Jain R, Jing R, Vunnam SR. 2020.

276 Gastrointestinal, hepatobiliary, and pancreatic manifestations of COVID-19. Journal of clinical

277 virology : the official publication of the Pan American Society for Clinical Virology,

278 128:104386.

279 Peng G, Sun D, Rajashankar KR, Qian Z, Holmes KV, Li F. 2011. Crystal structure of mouse 280 coronavirus receptor-binding domain complexed with its murine receptor. Proceedings of the

281 National Academy of Sciences of the United States of America, 108:10696-10701.

282 Peng G, Xu L, Lin YL, Chen L, Pasquarella JR, Holmes KV, Li F. 2012. Crystal structure of 283 bovine coronavirus spike protein lectin domain. The Journal of biological chemistry, 287:4193128441938.

285 Perricone C, Triggianese P, Bartoloni E, Cafaro G, Bonifacio AF, Bursi R, Perricone R, Gerli R. 286 2020. The anti-viral facet of anti-rheumatic drugs: Lessons from COVID-19. Journal of 287 Autoimmunity:102468.

288 Ren Z, Liang W, Sheng J, Xun C, Xu T, Cao R, Sheng W. 2019. Gal-3 is a potential biomarker 289 for spinal cord injury and Gal-3 deficiency attenuates neuroinflammation through 290 ROS/TXNIP/NLRP3 signaling pathway. Bioscience reports, 39:10.1042/BSR20192368. 
291

292

293

294

295

296

297

298

299

300

301

302

303

304

305

306

307

308

309

310

311

312

313

314

315

316

317

318

Saito S, Alkhatib A, Kolls JK, Kondoh Y, Lasky JA. 2019. Pharmacotherapy and adjunctive treatment for idiopathic pulmonary fibrosis (IPF). Journal of thoracic disease, 11:S1740-S1754.

Stegmayr J, Zetterberg F, Carlsson MC, Huang X, Sharma G, Kahl-Knutson B, Schambye H, Nilsson UJ, Oredsson S, Leffler H. 2019. Extracellular and intracellular small-molecule galectin3 inhibitors. Scientific reports, 9:2186-019-38497-8.

Tortorici MA, Walls AC, Lang Y, Wang C, Li Z, Koerhuis D, Boons GJ, Bosch BJ, Rey FA, de Groot RJ, Veesler D. 2019. Structural basis for human coronavirus attachment to sialic acid receptors. Nature structural \& molecular biology, 26:481-489.

Uchino Y, Woodward AM, Mauris J, Peterson K, Verma P, Nilsson UJ, Rajaiya J, Argueso P. 2018. Galectin-3 is an amplifier of the interleukin-1beta-mediated inflammatory response in corneal keratinocytes. Immunology, 154:490-499.

Wang Q, Zhang Y, Wu L, Niu S, Song C, Zhang Z, Lu G, Qiao C, Hu Y, Yuen KY, Wang Q, Zhou H, Yan J, Qi J. 2020. Structural and Functional Basis of SARS-CoV-2 Entry by Using Human ACE2. Cell.

Wang WH, Lin CY, Chang MR, Urbina AN, Assavalapsakul W, Thitithanyanont A, Chen YH, Liu FT, Wang SF. 2019. The role of galectins in virus infection - A systemic literature review. Journal of microbiology, immunology, and infection = Wei mian yu gan ran za zhi.

Whittaker A, Anson M, Harky A. 2020. Neurological Manifestations of COVID-19: A review. Acta Neurologica Scandinavica.

Wu G, Lu ZH, Andre S, Gabius HJ, Ledeen RW. 2016. Functional interplay between ganglioside GM1 and cross-linking galectin-1 induces axon-like neuritogenesis via integrin-based signaling and TRPC5-dependent $\mathrm{Ca}(2)(+)$ influx. Journal of neurochemistry, 136:550-563.

Zhai P, Ding Y, Wu X, Long J, Zhong Y, Li Y. 2020. The epidemiology, diagnosis and treatment of COVID-19. International journal of antimicrobial agents: 105955.

Zhou P, Yang XL, Wang XG, Hu B, Zhang L, Zhang W, Si HR, Zhu Y, Li B, Huang CL, Chen HD, Chen J, Luo Y, Guo H, Jiang RD, Liu MQ, Chen Y, Shen XR, Wang X, Zheng XS, Zhao K, Chen QJ, Deng F, Liu LL, Yan B, Zhan FX, Wang YY, Xiao GF, Shi ZL. 2020. A pneumonia outbreak associated with a new coronavirus of probable bat origin. Nature, 579:270-273. 
Figure 1

Structural similarities of SARS-CoV2 S1-NTD and human Gal-3.

The structural topologies of the (A) SARS-CoV2 S1-NTD (PDB ID: 6VXX) and (B) human Gal-3 (PDB ID: 1A3K) are shown as schematic illustrations, where $\beta$-strands are depicted as arrows and $\alpha$-helices as cylinders.

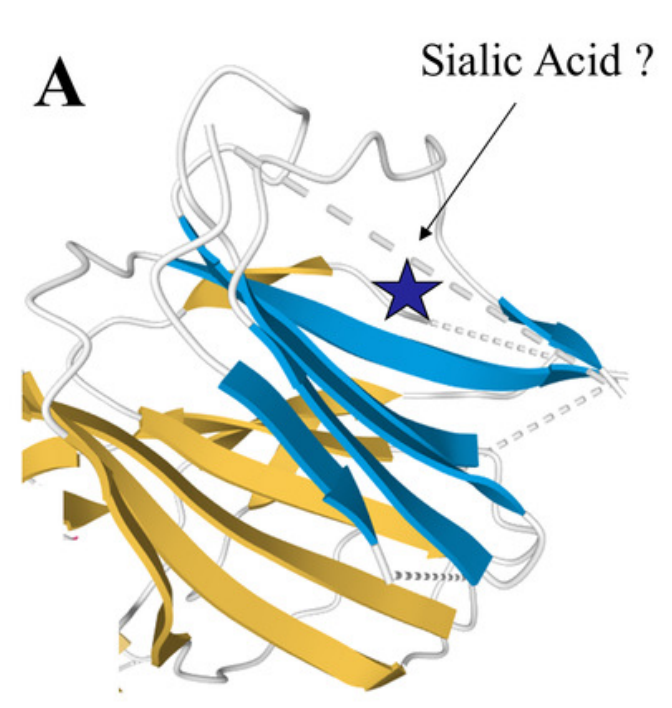

SARS-CoV2 S1-NTD

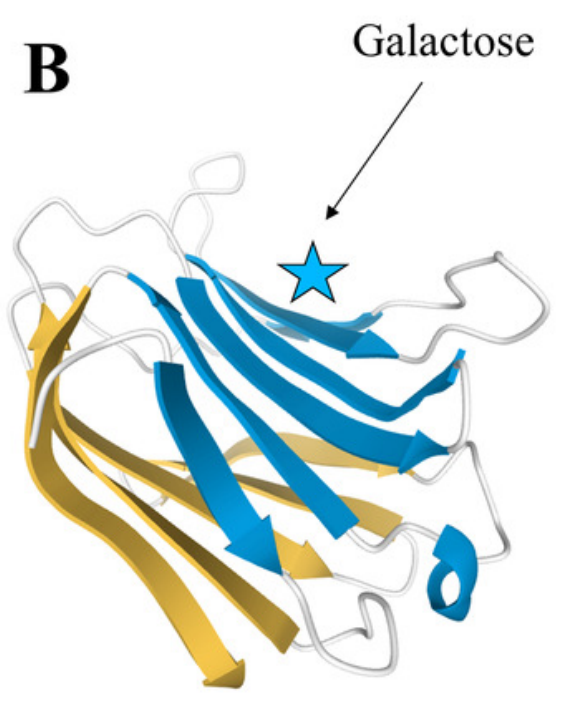

Human Gal-3 
Table $\mathbf{1}$ (on next page)

Receptors used for entry amongst $\beta$-coronaviridae 


\begin{tabular}{ccc}
\hline Virus & Receptor & NTD / CTD Mediated \\
\hline MHV & CEACAM1 & NTD mediated \\
\hline BCoV & Sialic Acids & NTD mediated \\
\hline MERS-CoV & DPP4 & CTD mediated \\
\hline SARS-CoV & ACE2 & CTD mediated \\
\hline SARS-CoV2 & ACE2 & CTD mediated \\
\hline
\end{tabular}

1 
Figure 2

Gal-3 inhibition may disrupt the attachment of SARS-CoV2 S1-NTD to GM1 gangliosides on the cell surface.

\section{SARS-CoV2}

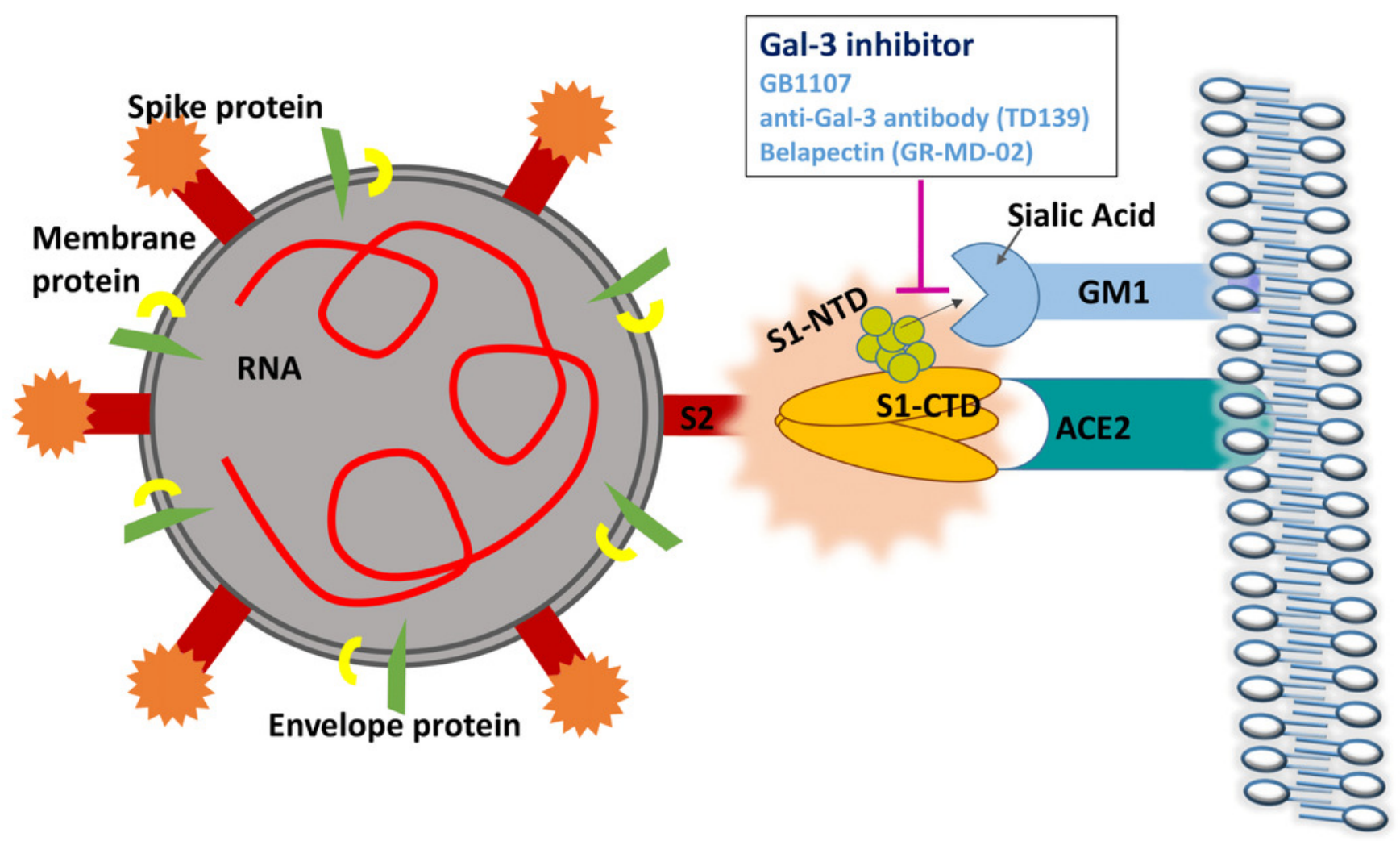


Figure 3

Gal-3 inhibition suppresses the release of IL- 6 and TNF- $\alpha$ from dendritic cells.

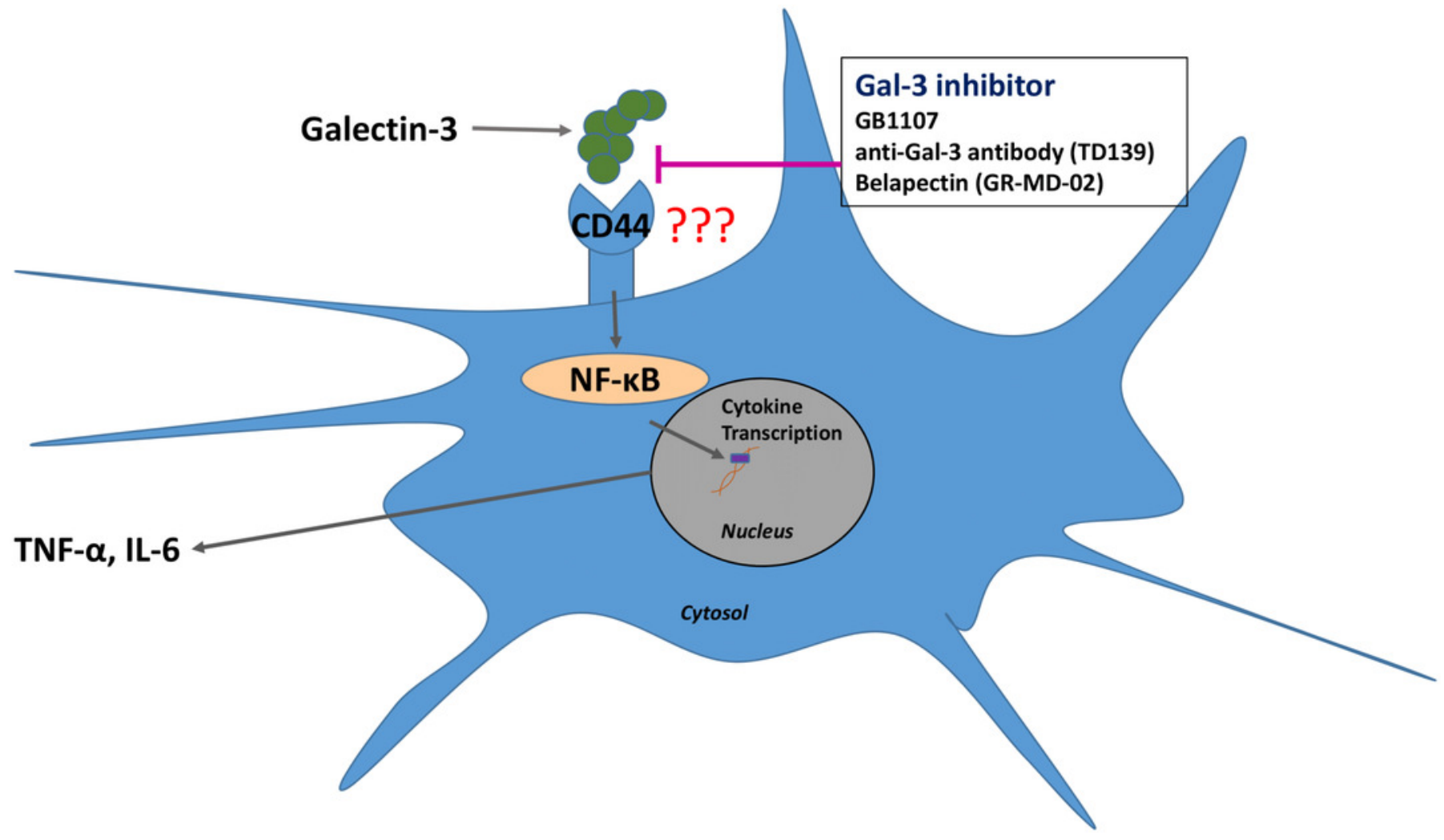

